\title{
Global Convergence of Curve Search Methods for Unconstrained Optimization
}

\author{
Zhiwei Xu${ }^{1}$, Yongning Tang ${ }^{2}$, Zhen-Jun Shi ${ }^{3}$ \\ ${ }^{1}$ Computer and Information Science, University of Michigan, Dearborn, MI, USA \\ ${ }^{2}$ School of Information Technology, Illinois State University, Normal, IL, USA \\ ${ }^{3}$ Mathematics and Computer Science, Central State University, Wilberforce, $\mathrm{OH}, \mathrm{USA}$ \\ Email: zwxu@umich.edu,ytang@ilstu.edu,zshi@centralstate.edu
}

Received 20 December 2015; accepted 25 April 2016; published 28 April 2016

Copyright (C) 2016 by authors and Scientific Research Publishing Inc.

This work is licensed under the Creative Commons Attribution International License (CC BY). http://creativecommons.org/licenses/by/4.0/

\section{(c) (i) Open Access}

\begin{abstract}
In this paper we propose a new family of curve search methods for unconstrained optimization problems, which are based on searching a new iterate along a curve through the current iterate at each iteration, while line search methods are based on finding a new iterate on a line starting from the current iterate at each iteration. The global convergence and linear convergence rate of these curve search methods are investigated under some mild conditions. Numerical results show that some curve search methods are stable and effective in solving some large scale minimization problems.
\end{abstract}

Keywords

Unconstrained Optimization, Curve Search Method, Global Convergence, Convergence Rate

\section{Introduction}

Line search method is an important and mature technique in solving an unconstrained minimization problem

$$
\min f(x), x \in R^{n} \text {, }
$$

where $R^{n}$ is an n-dimensional Euclidean space and $f: R^{n} \rightarrow R^{1}$ is a continuously differentiable function. It takes the form

$$
x_{k+1}=x_{k}+\alpha_{k} d_{k}, k=0,1,2, \cdots,
$$

where $d_{k}$ is a descent direction of $f(x)$ at $x_{k}$ and $\alpha_{k}$ is a step size to satisfy the descent condition

$$
f\left(x_{k}+\alpha_{k} d_{k}\right)<f\left(x_{k}\right), k=0,1,2, \cdots
$$


One hopes that $\left\{x_{k}\right\}$ generated by line search method converges to the minimizer $x^{*}$ of (1) in some sense. Let $x_{k}$ be the current iterate. We denote $f\left(x_{k}\right)$ by $f_{k}, \nabla f\left(x_{k}\right)$ by $g_{k}$, and $f\left(x^{*}\right)$ by $f^{*}$, respectively.

At the $k$-th iteration of line search methods, one first chooses a search direction and then seeks a step size along the search direction and completes one iteration (see [1]). The search direction $d_{k}$ is generally required to satisfy

$$
g_{k}^{T} d_{k}<0
$$

which guarantees that $d_{k}$ is a descent direction of $f(x)$ at $x_{k}$ (e.g. [2] [3]). In order to guarantee the global convergence, we sometimes require $d_{k}$ to satisfy the sufficient descent condition

$$
g_{k}^{T} d_{k} \leq-c\left\|g_{k}\right\|^{2},
$$

where $c>0$ is a constant. Moreover, the angle testing condition is commonly used in proving the global convergence of related line search methods, that is

$$
\cos \left\langle-g_{k}, d_{k}\right\rangle=-\frac{g_{k}^{T} d_{k}}{\left\|g_{k}\right\| \cdot\left\|d_{k}\right\|} \geq \eta,
$$

where $1 \geq \eta>0$.

In line search methods we try to find an $\alpha$ to reduce $f(x)$ over the ray $S_{k}=\left\{x_{k}+\alpha d_{k} \mid \alpha>0\right\}$ at the $k$ th iteration, while curve search method is to define the next iterate on the curve

$$
S_{k}=\left\{y_{k}(\alpha) \mid y_{k}: R_{+}^{1} \rightarrow R^{n}, y_{k}(\alpha) \in C^{1}, y_{k}(0)=x_{k}, y_{k}^{\prime}(0)=d_{k}\right\},
$$

where $R_{+}^{n}=[0,+\infty)$ and $y_{k}(\alpha) \in C^{1}$ means that $y_{k}(\alpha)$ is continuously differentiable on $[0,+\infty)$. It is obvious that line search method is a special one of curve search methods. In other words, curve search method is a generalization of line search methods.

McCormick [4] and Israel Zang [5] proposed an arc method for mathematical programming, which is actually a special one of curve search methods. Similarly as in line search methods, how to choose a curve at each iteration is the key to using curve search methods.

Botsaris [6]-[9] studied differential gradient method (abbreviated as ODE method) for unconstrained minimization problems. It is required to solve differential equations at the $k$-th iteration

$$
\frac{\mathrm{d} x}{\mathrm{~d} t}=-g(x), x(0)=x_{k},
$$

or to solve

$$
\frac{\mathrm{d} x}{\mathrm{~d} t}=-H(x) g(x), x(0)=x_{k},
$$

where $H(x)=\nabla^{2} f(x)^{-1}$ and $g(x)=\nabla f(x)$. The ODE method has been investigated by many researchers (e.g. [10]-[14]) and is essentially a curve search method.

However, it is required to solve some initial-value problems of ordinary differential equations to define the curves in ODE methods. Some other curve search methods with memory gradient have also been investigated and been proved to be a kind of promising methods for large-scale unconstrained optimization problems (see [15] [16]). Other literature on curve search methods have appeared in the literature [17]-[19]. To the best of our knowledge, the unified form of curve search methods has rarely been studied in present literature. It is necessary to study the general scheme of curve search methods and its global convergence.

In this paper we present a new family of curve search methods for unconstrained minimization problems and prove their global convergence and linear convergence rate under some mild conditions. These method are based on searching a new iterate along a curve at each iteration, while line search methods are based on finding a new iterate on a line starting from the current iterate at each iteration. Many curve search rules proposed in the paper can guarantee the global convergence and linear convergence rate of these curve search methods. Some implementable version of curve search methods are presented and numerical results show that some curve search methods are stable, useful and efficient in solving large scale minimization problems.

The rest of this paper is organized as follows. In the next section we describe the curve search methods. In 
Sections 3 and 4 we analyze its global convergence and linear convergence rate respectively. In Section 5 we report some techniques for choosing the curves and conduct some numerical experiments. And finally some conclusion remarks are given in Section 6.

\section{Curve Search Method}

We first assume that

(H1). The objective function $f(x)$ is continuously differentiable on $R^{n}$ and the level set $L\left(x_{0}\right)=\left\{x \in R^{n} \mid f(x) \leq f\left(x_{0}\right)\right\}$ is bounded, where $x_{0}$ is given.

(H1'). The gradient $g(x)=\nabla f(x)$ of $f(x)$ is Lipschitz continuous on an open bounded convex set $B$ that contains the level set $L\left(x_{0}\right)$, i.e., there exists $M^{\prime}$ such that

$$
\|g(x)-g(y)\| \leq M^{\prime}\|x-y\|, \forall x, y \in B .
$$

Definition 2.1. Let $x_{k}$ be the current iterate and $B$ be an open bounded convex set that contains $L\left(x_{0}\right)$. We define a curve within B through $x_{k}$ as follows

$$
y=y_{k}(\alpha): R_{+}^{1} \rightarrow R^{n}
$$

where $R_{+}^{1}=[0,+\infty)$ and $y_{k}(\alpha)$ is continuously differentiable on $R_{+}^{1}$ with $y_{k}(0)=x_{k}$ and $y_{k}^{\prime}(0)=d_{k}$.

Definition 2.2. We call the one-dimensional function $\gamma: R_{+}^{1} \rightarrow R_{+}^{1}$ a forcing function if

$$
\gamma(\alpha) \rightarrow 0 \text { as } \alpha \rightarrow 0
$$

where $R_{+}^{1}=[0,+\infty)$.

It is obvious that the addition, the multiplication and the composite function of two forcing functions are also forcing functions.

In order to guarantee the global convergence of curve search methods, we suppose that the initial descent direction $d_{k}$ and the curve $y_{k}(\alpha)$ satisfies the following assumption.

(H2). The search curve sequence $\left\{y_{k}(\alpha)\right\}$ satisfies

$$
\begin{aligned}
& \left\|y_{k}(\alpha)-y_{k}(0)\right\| \leq \gamma_{1}(\alpha), \forall k ; \\
& \frac{\left\|y_{k}^{\prime}(\alpha)-y_{k}^{\prime}(0)\right\|}{\left\|y_{k}^{\prime}(0)\right\|} \leq \gamma_{2}(\alpha), \forall k,
\end{aligned}
$$

where $\gamma_{1}: R_{+}^{1} \rightarrow R_{+}^{1}$ and $\gamma_{2}: R_{+}^{1} \rightarrow R_{+}^{1}$ are forcing functions.

Remark 1. In fact, if there exist $l$ and $l^{\prime}$ such that $y_{k}(0)=x_{k}$, and

$$
\left\|y_{k}(\alpha)-y_{k}(0)\right\| \leq l \alpha^{p}, \frac{\left\|y_{k}^{\prime}(\alpha)-y_{k}^{\prime}(0)\right\|}{\left\|y_{k}^{\prime}(0)\right\|} \leq l^{\prime} \alpha^{q}, \forall k \geq 0,
$$

where $p, q \in(0,+\infty)$, then the curve sequence $\left.\left\{y_{k}(\alpha)\right)\right\}$ satisfies $(H 2)$.

This kind of curves are easy to find. For example,

$$
S_{k}=\left\{y_{k}(\alpha) \mid y_{k}(\alpha)=x_{k}-\alpha g_{k}-\alpha^{2}\left\|g_{k}\right\| g_{k-1}\right\}
$$

are curves that satisfy $(H 2)$ and so are the following curves

$$
S_{k}=\left\{y_{k}(\alpha) \mid y_{k}(\alpha)=x_{k}+\alpha d_{k}-\alpha^{2}\left\|d_{k}\right\| d_{k-1}\right\}
$$

(for $k \geq 1$ ), provided that $\left\{g_{k}\right\}$ and $\left\{d_{k}\right\}$ are bounded for all $k \geq 0$.

Remark 2. If $x=y_{k}(\alpha)$ is twice continuously differentiable and there exist $M$ and $\bar{M}$ such that $\left\|y_{k}^{\prime}(\alpha)\right\| \leq M$ and $\left\|y_{k}^{\prime \prime}(\alpha)\right\| \leq \bar{M}\left\|y_{k}^{\prime}(0)\right\|$ for all $k \geq 0$, then the curve sequence $\left\{y_{k}(\alpha)\right\}$ satisfies $(H 2)$ because of

$$
\left\|y_{k}(\alpha)-y_{k}(0)\right\| \leq \alpha M \rightarrow 0(\alpha \rightarrow 0, \forall k)
$$

and 


$$
\frac{\left\|y_{k}^{\prime}(\alpha)-y_{k}^{\prime}(0)\right\|}{\left\|y_{k}^{\prime}(0)\right\|} \leq \alpha \bar{M} \rightarrow 0(\alpha \rightarrow 0, \forall k) .
$$

Remark 3. In line search methods, if we let $y_{k}(\alpha)=x_{k}+\alpha d_{k}$ and $\left\{\left\|d_{k}\right\|\right\}$ be bounded for all $\mathrm{k}$, then $\left\{y_{k}(\alpha)\right\}$ satisfies (H2). As a result, line search method is a special one of curve search methods and its convergence can be derived from the convergence of curve search methods.

In the sequel, we describe the curve search method.

Algorithm (A).

Step 0. Choose $x_{0} \in R^{n}$ and set $k:=0$.

Step 1. If $\left\|g_{k}\right\|=0$ then stop else go to Step 2;

Step 2. Let $y_{k}(\alpha)$ be defined by Definition 2.1 and $y_{k}^{\prime}(0)=d_{k}$ satisfies (4). Set $x_{k+1}=y_{k}\left(\alpha_{k}\right)$ where $\alpha_{k}$ is selected by some curve search rule;

Step 3. Set $k:=k+1$ and go to Step 1 .

Once the initial descent direction $d_{k}$ and the search curve $y_{k}(\alpha)$ are determined at the $k$-th iteration, we need to seek a step size $\alpha_{k}$ such that

$$
f\left(y_{k}\left(\alpha_{k}\right)\right)<f_{k} .
$$

For convenience, let $d_{k}=y_{k}^{\prime}(0)$ satisfy (4). There are several curve search rules as follows.

(a) Exact Curve Search Rule. Select an $\alpha_{k}$ to satisfy

$$
f\left(y_{k}\left(\alpha_{k}\right)\right)=\min _{\alpha>0} f\left(y_{k}(\alpha)\right) \text {. }
$$

(b) Approximate Exact Curve Search Rule. Select $\alpha_{k}$ to satisfy

$$
\alpha_{k}=\arg \min \left\{\alpha \mid g\left(y_{k}(\alpha)\right)^{T} y_{k}^{\prime}(\alpha)=0\right\} .
$$

(c) Armijo-type Curve Search Rule. Set $L>0, \sigma \in\left(0, \frac{1}{2}\right), \rho \in(0,1)$ and $s_{k}=-\frac{g_{k}^{T} d_{k}}{L\left\|d_{k}\right\|^{2}}$. Choose $\alpha_{k}$ to be the largest $\alpha$ in $\left\{s_{k}, \rho s_{k}, \rho^{2} s_{k}, \cdots\right\}$ such that

$$
f_{k}-f\left(y_{k}(\alpha)\right) \geq-\sigma \alpha g_{k}^{T} d_{k} \text {. }
$$

(d) Limited Exact Curve Search Rule. Set $L>0$ and $s_{k}=-\frac{g_{k}^{T} d_{k}}{L\left\|d_{k}\right\|^{2}}$. Choose $\alpha_{k}$ to satisfy

$$
f\left(y_{k}\left(\alpha_{k}\right)\right)=\min _{\alpha \in\left[0, s_{k}\right]} f\left(y_{k}(\alpha)\right) \text {. }
$$

(e) Goldstein-type Curve Search Rule. Set $\sigma \in\left(0, \frac{1}{2}\right)$ Choose $\alpha_{k}$ to satisfy

$$
(1-\sigma) \alpha_{k} g_{k}^{T} d_{k} \leq f\left(y_{k}\left(\alpha_{k}\right)\right)-f_{k} \leq \sigma \alpha_{k} g_{k}^{T} d_{k}
$$

(f) Strong Wolfe-type Curve Search Rule. Set $\sigma \in\left(0, \frac{1}{2}\right)$ and $\beta \in(\sigma, 1)$. Choose $\alpha_{k}$ to satisfy simultaneously

$$
f\left(y_{k}\left(\alpha_{k}\right)\right)-f_{k} \leq \sigma \alpha_{k} g_{k}^{T} d_{k},
$$

and

$$
\left|g\left(y_{k}\left(\alpha_{k}\right)\right)^{T} y_{k}^{\prime}\left(\alpha_{k}\right)\right| \leq-\beta g_{k}^{T} d_{k} .
$$

(g) Wolfe-type Curve Search Rule. Set $\sigma \in\left(0, \frac{1}{2}\right)$ and $\beta \in(\sigma, 1)$. Choose $\alpha_{k}$ to satisfy simultaneously 
(12) and

$$
g\left(y_{k}\left(\alpha_{k}\right)\right)^{T} y_{k}^{\prime}\left(\alpha_{k}\right) \geq-\beta g_{k}^{T} d_{k} .
$$

Lemma 2.1. Let $y_{k}(\alpha)$ be defined in Definition 2.1 and $d_{k}=y_{k}^{\prime}(0)$ satisfies (4). Assumptions (H1) and (H2) hold and let $\phi_{k}(\alpha)=f\left(y_{k}(\alpha)\right)$. Then

$$
\frac{\left|\phi_{k}^{\prime}(\alpha)-\phi_{k}^{\prime}(0)\right|}{\left\|y_{k}^{\prime}(0)\right\|} \leq \gamma_{3}(\alpha), \forall k,
$$

where $\gamma_{3}(\alpha)$ is a forcing function.

Proof. Assumption (H1) and Definition 2.1 imply that $g(x)$ is uniformly continuous on B and thus, there exist $M_{0}$ and a forcing function $\gamma_{4}(\alpha)$ such that

$$
\left\|g\left(y_{k}(\alpha)\right)\right\| \leq M_{0},\left\|g\left(y_{k}(\alpha)\right)-g\left(y_{k}(0)\right)\right\| \leq \gamma_{4}\left(\left\|y_{k}(\alpha)-y_{k}(0)\right\|\right), \forall k
$$

By (H2), Definition 2.1 and (15), noting that $y_{k}^{\prime}(0)=d_{k}$ and $g\left(y_{k}(0)\right)=g_{k}$, we have

$$
\begin{aligned}
& \frac{\left|\phi_{k}^{\prime}(\alpha)-\phi_{k}^{\prime}(0)\right|}{\left\|y_{k}^{\prime}(0)\right\|}=\frac{\left|g\left(y_{k}(\alpha)\right)^{T} y_{k}^{\prime}(\alpha)-g\left(y_{k}(0)\right)^{T} y_{k}^{\prime}(0)\right|}{\| y_{k}^{\prime}(0) \mid} \\
& \leq \frac{\left|g\left(y_{k}(\alpha)\right)^{T} y_{k}^{\prime}(\alpha)-g\left(y_{k}(\alpha)\right)^{T} y_{k}^{\prime}(0)\right|}{\left\|y_{k}^{\prime}(0)\right\|}+\frac{\left|g\left(y_{k}(\alpha)\right)^{T} y_{k}^{\prime}(0)-g\left(y_{k}(0)\right)^{T} y_{k}^{\prime}(0)\right|}{\left\|y_{k}^{\prime}(0)\right\|} \\
& =\frac{\left|g\left(y_{k}(\alpha)\right)^{T}\left[y_{k}^{\prime}(\alpha)-y_{k}^{\prime}(0)\right]\right|}{\left\|y_{k}^{\prime}(0)\right\|}+\frac{\left|\left[g\left(y_{k}(\alpha)\right)-g\left(y_{k}(0)\right)\right]^{T} y_{k}^{\prime}(0)\right|}{\left\|y_{k}^{\prime}(0)\right\|} \\
& \leq \frac{\left\|g\left(y_{k}(\alpha)\right)\right\| \cdot\left\|y_{k}^{\prime}(\alpha)-y_{k}^{\prime}(0)\right\|}{\left\|y_{k}^{\prime}(0)\right\|}+\frac{\left\|g\left(y_{k}(\alpha)\right)-g\left(y_{k}(0)\right)\right\| \cdot\left\|y_{k}^{\prime}(0)\right\|}{\left\|y_{k}^{\prime}(0)\right\|} \\
& \leq\left\|g\left(y_{k}(\alpha)\right)\right\| \cdot \gamma_{2}(\alpha)+\gamma_{4}\left(\left\|y_{k}(\alpha)-y_{k}(0)\right\|\right) \\
& \leq M_{0} \gamma_{2}(\alpha)+\gamma_{4}\left(\gamma_{1}(\alpha)\right) \\
& =\gamma_{3}(\alpha) .
\end{aligned}
$$

Lemma 2.2. If (H1) holds and $y_{k}(\alpha)$ is defined by Definition 2.1 and $d_{k}=y_{k}^{\prime}(0)$ satisfies (4), then $\alpha_{k}$ is well defined in the seven curve search rules.

Proof. Let $\phi_{k}(\alpha)=f\left(y_{k}(\alpha)\right)$. Obviously $\phi_{k}(0)=f_{k}, \phi_{k}^{\prime}(0)=g_{k}^{T} d_{k}<0$. The following limit

$$
\lim _{\alpha \rightarrow+0} \frac{\phi_{k}(0)-\phi_{k}(\alpha)}{\alpha}=-\phi_{k}^{\prime}(0)>-\sigma \phi_{k}^{\prime}(0)
$$

implies that there exists $\alpha_{k}^{\prime}>0$ such that

$$
\frac{\phi_{k}(0)-\phi_{k}(\alpha)}{\alpha} \geq-\sigma \phi_{k}^{\prime}(0), \forall \alpha \in\left[0, \alpha_{k}^{\prime}\right] .
$$

Thus

$$
f_{k}-f\left(y_{k}(\alpha)\right)=\phi_{k}(0)-\phi_{k}(\alpha) \geq-\sigma \alpha \phi_{k}^{\prime}(0)=-\sigma \alpha g_{k}^{T} d_{k}, \forall \alpha \in\left[0, \alpha_{k}^{\prime}\right],
$$

which shows that the curve search rules (a), (b), (c) and (d) are well-defined.

In the following we prove that the curve search rules (e), (f) and (g) are also well-defined.

For the curve search rule (e), (H1) and

$$
\lim _{\alpha \rightarrow+\infty}(\sigma \alpha) g_{k}^{T} d_{k}=-\infty
$$


imply that the curve $z=f\left(y_{k}(\alpha)\right)-f_{k}$ and the line $z=\sigma \alpha g_{k}^{T} d_{k}$ must have an intersection point $\left(\alpha_{k}, z_{k}\right)$ and thus

$$
(1-\sigma) \alpha_{k} g_{k}^{T} d_{k} \leq f\left(y_{k}\left(\alpha_{k}\right)\right)-f_{k}=\sigma \alpha_{k} g_{k}^{T} d_{k}=z_{k},
$$

which shows that the curve search rule (e) is well-defined.

For the curve search rules (f) and (g), (H1) and

$$
\lim _{\alpha \rightarrow+\infty}(\sigma \alpha) g_{k}^{T} d_{k}=-\infty
$$

imply that the curve $z=f\left(y_{k}(\alpha)\right)-f_{k}$ and the line $z=\alpha \sigma g_{k}^{T} d_{k}$ must have an intersection point and suppose that $\left(\alpha_{k}^{\prime}, z_{k}^{\prime}\right)$ is not the origin $(0,0)$ but the nearest intersection point to $(0,0)$. Thus

$$
f\left(y_{k}\left(\alpha_{k}^{\prime}\right)\right)-f_{k}=\alpha_{k}^{\prime} \sigma g_{k}^{T} d_{k},
$$

and

$$
f\left(y_{k}\left(\theta \alpha_{k}^{\prime}\right)\right)-f_{k} \leq \theta \alpha_{k}^{\prime} \sigma g_{k}^{T} d_{k},
$$

where $\theta \in[0,1]$. Using the mean value theorem, there exists $\theta_{k} \in[0,1]$ such that

$$
f\left(y_{k}\left(\alpha_{k}^{\prime}\right)\right)-f_{k}=\alpha_{k}^{\prime} g\left(y_{k}\left(\theta_{k} \alpha_{k}^{\prime}\right)\right)^{T} y_{k}^{\prime}\left(\theta_{k} \alpha_{k}^{\prime}\right) .
$$

By (16) we have

$$
\alpha_{k}^{\prime} g\left(y_{k}\left(\theta_{k} \alpha_{k}^{\prime}\right)\right)^{T} y_{k}^{\prime}\left(\theta_{k} \alpha_{k}^{\prime}\right)=\alpha_{k}^{\prime} \sigma g_{k}^{T} d_{k}
$$

and thus,

$$
g\left(y_{k}\left(\theta_{k} \alpha_{k}^{\prime}\right)\right)^{T} y_{k}^{\prime}\left(\theta_{k} \alpha_{k}^{\prime}\right)=\sigma g_{k}^{T} d_{k} \geq \beta g_{k}^{T} d_{k}
$$

Therefore,

$$
g\left(y_{k}\left(\theta_{k} \alpha_{k}^{\prime}\right)\right)^{T} y_{k}^{\prime}\left(\theta_{k} \alpha_{k}^{\prime}\right) \geq \beta g_{k}^{T} d_{k} .
$$

Obviously, it follows from (17) and (18) that $\alpha_{k}=\theta_{k} \alpha_{k}^{\prime}$ satisfies (12) and (14) (also satisfies (12) and (13)). This shows that the curve search rules (f) and (g) are well-defined.

\section{Global Convergence}

Theorem 3.1. Assume that (H1), $\left(H 1^{\prime}\right)$ and $(H 2)$ hold, $y_{k}(\alpha)$ is defined by Definition 2.1 and $d_{k}=y_{k}^{\prime}(0)$ satisfies (5) and

$$
\left\|d_{k}\right\| \leq c_{0}\left\|g_{k}\right\|,
$$

where $c_{0}>0$ is a constant. If $\alpha_{k}$ is defined by the curve search rules (a), (b), (c) or (d) and Algorithm (A) generates an infinite sequence $\left\{x_{k}\right\}$, then

$$
\lim _{k \rightarrow+\infty}\left\|g_{k}\right\|=0 \text {. }
$$

Proof. Using reduction to absurdity, suppose that there exist an infinite subset $K \subseteq\{1,2,3, \cdots$,$\} and an$ $\epsilon>0$ such that

$$
\left\|g_{k}\right\| \geq \epsilon, \quad \forall k \in K
$$

(H1) implies that $\left\{\left\|g_{k}\right\|\right\}$ has a bound, say $M_{0}$, i.e., $\left\|g_{k}\right\| \leq M_{0}, \forall k$, and thus $\left\|d_{k}\right\| \leq c_{0} M_{0}, \forall k$.

Let

$$
\eta_{0}=\inf _{\forall k \in K}\left\{\alpha_{k}\right\}
$$

In the case of $\eta_{0}>0$, for the curve search rule (c), there must exist $\eta_{0}^{\prime}>0$ such that 


$$
\alpha_{k}>\eta_{0}^{\prime} s_{k}, k \in K
$$

because of

$$
s_{k}=-\frac{g_{k}^{T} d_{k}}{L \|\left. d_{k}\right|^{2}} \geq \frac{c\left\|g_{k}\right\|^{2}}{L\left\|d_{k}\right\|^{2}} \geq \frac{c}{c_{0}^{2} L}>0 .
$$

By (9) and (21) we have

$$
\begin{aligned}
f_{k}-f\left(y_{k}\left(\alpha_{k}\right)\right) & \geq-\alpha_{k} \sigma g_{k}^{T} d_{k} \geq-\eta_{0}^{\prime} \sigma s_{k} g_{k}^{T} d_{k}=\frac{\eta_{0}^{\prime} \sigma}{L}\left(-\frac{g_{k}^{T} d_{k}}{\left\|d_{k}\right\|}\right)^{2} \\
& \geq \frac{\eta_{0}^{\prime} \sigma}{L}\left(\frac{c\left\|g_{k}\right\|^{2}}{\left\|d_{k}\right\|}\right)^{2} \geq \frac{c^{2} \eta_{0}^{\prime} \sigma}{L c_{0}^{2}}\left\|g_{k}\right\|^{2}, k \in K .
\end{aligned}
$$

By (H1) we can obtain

$$
\lim _{k \in K, k \rightarrow \infty}\left\|g_{k}\right\|^{2}=0
$$

which contradicts (20).

In the case of $\eta_{0}=0$, there must exist an infinite subset $K_{1} \subseteq K$ such that

$$
\lim _{k \in K_{1}, k \rightarrow \infty} \alpha_{k}=0 .
$$

Therefore, for sufficiently large $k \in K_{1}, \alpha_{k}<s_{k}$ implies that $\alpha / \rho \leq s_{k}$ and

$$
f_{k}-f\left(y_{k}\left(\alpha_{k} / \rho\right)\right)<-\sigma\left(\alpha_{k} / \rho\right) g_{k}^{T} d_{k} .
$$

Using the mean value theorem on the left-hand side of the above inequality, there exists $\theta_{k} \in[0,1]$ such that

$$
f_{k}-f\left(y_{k}\left(\alpha_{k} / \rho\right)\right)=-\left(\alpha_{k} / \rho\right) g\left(y_{k}\left(\theta_{k} \alpha_{k} / \rho\right)\right)^{T} y_{k}^{\prime}\left(\theta_{k} \alpha_{k} / \rho\right),
$$

and thus

$$
-\left(\alpha_{k} / \rho\right) g\left(y_{k}\left(\theta_{k} \alpha_{k} / \rho\right)\right)^{T} y_{k}^{\prime}\left(\theta_{k} \alpha_{k} / \rho\right)<-\sigma\left(\alpha_{k} / \rho\right) g_{k}^{T} d_{k}
$$

Hence

$$
g\left(y_{k}\left(\theta_{k} \alpha_{k} / \rho\right)\right)^{T} y_{k^{\prime}}\left(\theta_{k} \alpha_{k} / \rho\right)>\sigma g_{k}^{T} d_{k}, k \in K_{1} .
$$

By (22), (23) and Lemma 2.1, we have

$$
\begin{aligned}
\frac{c(1-\sigma)}{c_{0} M_{0}}\left\|g_{k}\right\|^{2} & \leq-(1-\sigma) \frac{g_{k}^{T} d_{k}}{\left\|d_{k}\right\|} \\
& <\frac{g\left(y_{k}\left(\theta_{k} \alpha_{k} / \rho\right)\right)^{T} y_{k}^{\prime}\left(\theta_{k} \alpha_{k} / \rho\right)-g_{k}^{T} d_{k}}{\left\|d_{k}\right\|} \\
& \leq \frac{\left|\phi_{k}^{\prime}\left(\theta_{k} \alpha_{k} / \rho\right)-\phi_{k}^{\prime}(0)\right|}{\left\|d_{k}\right\|} \leq \gamma_{3}\left(\theta_{k} \alpha_{k} / \rho\right) \\
& \rightarrow 0\left(k \in K_{1}, k \rightarrow \infty\right),
\end{aligned}
$$

which also contradicts (20).

In fact, we can prove that $\eta_{0}>0$ for the curve search rule (c). If $\eta_{0}=0$ then there exists an infinite subset $K_{1}$ such that (22) holds and thus, (23) holds. By (19), (5), (H1'), the mean value theorem, (H2) and (22), we have 


$$
\begin{aligned}
\frac{c(1-\sigma)}{c_{0}^{2}} & \leq \frac{c(1-\sigma)\left\|g_{k}\right\|^{2}}{c_{0}^{2}\left\|g_{k}\right\|^{2}} \leq \frac{c(1-\sigma)\left\|g_{k}\right\|^{2}}{\left\|d_{k}\right\|^{2}} \leq-\frac{(1-\sigma) g_{k}^{T} d_{k}}{\left\|d_{k}\right\|^{2}} \leq \frac{\phi_{k}^{\prime}\left(\alpha_{k} \theta_{k} / \rho\right)-\phi_{k}^{\prime}(0)}{\left\|d_{k}\right\|^{2}} \\
& =\frac{\left[g\left(y_{k}\left(\alpha_{k} \theta_{k} / \rho\right)\right)-g_{k}\right]^{T} y_{k}^{\prime}\left(\alpha_{k} \theta_{k} / \rho\right)}{\left\|d_{k}\right\|^{2}}+\frac{g_{k}^{T}\left[y_{k}^{\prime}\left(\alpha_{k} \theta_{k} / \rho\right)-d_{k}\right]}{\left\|d_{k}\right\|^{2}} \\
& \leq \frac{M^{\prime}\left\|y_{k}\left(\alpha_{k} \theta_{k} / \rho\right)-y_{k}(0)\right\| \cdot\left\|y_{k}^{\prime}\left(\alpha_{k} \theta_{k} / \rho\right)\right\|}{\left\|d_{k}\right\|^{2}}+\frac{\left.\left\|g_{k}\right\| \cdot \| y_{k}^{\prime}\left(\alpha_{k} \theta_{k} / \rho\right)-d_{k}\right] \|}{\left\|d_{k}\right\|^{2}} \\
& \leq \frac{\left.\left(\alpha_{k} \theta_{k} / \rho\right) \| \int_{0}^{1} y_{k}^{\prime}\left(t \alpha_{k} \theta_{k} / \rho\right) \mathrm{d} t\right)\|\cdot\| y_{k}^{\prime}\left(\alpha_{k} \theta_{k} / \rho\right) \|}{\left\|d_{k}\right\|^{2}}+\frac{\left.\| y_{k}^{\prime}\left(\alpha_{k} \theta_{k} / \rho\right)-d_{k}\right] \|}{c\left\|d_{k}\right\|} \\
& \rightarrow 0\left(k \in K_{1}, k \rightarrow \infty\right) .
\end{aligned}
$$

This contradiction shows that $\eta_{0}>0$.

For the curve search rules (a), (b) and (d), since $\eta_{0}>0$ for the curve search rule (c), let $\alpha_{k}$ be the step size defined by the three curve search rules (a), (b) and (d), and let $\alpha_{k}^{\prime}$ be the step size defined by the curve search rule (c), then we have

$$
f_{k}-f\left(y_{k}\left(\alpha_{k}\right)\right) \geq f_{k}-f\left(y_{k}\left(\alpha_{k}^{\prime}\right)\right) \geq \frac{\eta_{0}^{\prime} \sigma}{L}\left(-\frac{g_{k}^{T} d_{k}}{\left\|d_{k}\right\|}\right)^{2}, \forall k .
$$

This and (H1) imply that

$$
\frac{c}{c_{0}}\left\|g_{k}\right\| \leq \lim _{k \in K, k \rightarrow \infty}\left(-\frac{g_{k}^{T} d_{k}}{\left\|d_{k}\right\|}\right)=0
$$

holds for the curve search rules (a), (b) and (d), which contradicts (20). The conclusion is proved.

Theorem 3.2. Assume that $(H 1)$ and $(H 2)$ hold, $y_{k}(\alpha)$ is defined by Definition 2.1 and $d_{k}=y_{k}^{\prime}(0)$ satisfies (5) and (19), $\alpha_{k}$ is defined by the curve search rules (e), (f) or (g). Algorithm (A) generates an infinite sequence $\left\{x_{k}\right\}$. Then

$$
\lim _{k \rightarrow+\infty}\left\|g_{k}\right\|=0 .
$$

Proof. Using reduction to absurdity, suppose that there exist an infinite subset $K \subseteq\{0,1,2,3, \cdots\}$ and an $\epsilon>0$ such that (20) holds and let

$$
\eta_{0}=\inf _{\forall k \in K}\left\{\alpha_{k}\right\} .
$$

For the curve search rules (e), (f) and (g), in the case of $\eta_{0}>0$, by (11), (12) and (5), we have

$$
f_{k}-f\left(y_{k}\left(\alpha_{k}\right)\right) \geq-\alpha_{k} \sigma g_{k}^{T} d_{k} \geq \eta_{0} \sigma c\left\|g_{k}\right\|^{2}, k \in K \text {. }
$$

By (H1) we have

$$
\lim _{k \in K, k \rightarrow \infty}\left\|g_{k}\right\|^{2}=0
$$

which contradicts (20).

In the case of $\eta_{0}=0$, there must exist an infinite subset $K_{1} \subseteq K$ such that (22) holds. For the curve search rule (e), by the left-hand side inequality of (11) and using the mean value theorem, there exists $\theta_{k} \in[0,1]$ such that

$$
\phi_{k}\left(\alpha_{k}\right)=f\left(y_{k}\left(\alpha_{k}\right)\right)-f_{k}=\alpha_{k} g\left(y_{k}\left(\alpha_{k} \theta_{k}\right)\right)^{T} y_{k}^{\prime}\left(\theta_{k} \alpha_{k}\right) \geq(1-\sigma) \alpha_{k} g_{k}^{T} d_{k}
$$

Thus 


$$
\phi_{k}^{\prime}\left(\theta_{k} \alpha_{k}\right)=g\left(y_{k}\left(\alpha_{k} \theta_{k}\right)\right)^{T} y_{k}^{\prime}\left(\theta_{k} \alpha_{k}\right) \geq(1-\sigma)_{k}^{T} d_{k} .
$$

By (22), (24) and Lemma 2.1, we have

$$
-\frac{\sigma g_{k}^{T} d_{k}}{\left\|d_{k}\right\|} \leq \frac{\left|\phi_{k}^{\prime}\left(\theta_{k} \alpha_{k}\right)-\phi_{k}^{\prime}(0)\right|}{\left\|d_{k}\right\|} \leq \gamma_{3}\left(\theta_{k} \alpha_{k}\right) \rightarrow 0\left(k \in K_{1}, k \rightarrow \infty\right),
$$

which contradicts (20). For the curve search rules (f) and (g), by (14), (22) and Lemma 2.1, we have

$$
-(1-\beta) \frac{g_{k}^{T} d_{k}}{\left\|d_{k}\right\|} \leq \frac{\left|\phi_{k}^{\prime}\left(\alpha_{k}\right)-\phi_{k}^{\prime}(0)\right|}{\left\|d_{k}\right\|} \leq \gamma_{3}\left(\alpha_{k}\right) \rightarrow 0\left(k \in K_{1}, k \rightarrow \infty\right),
$$

which also contradicts (20).

The conclusions are proved.

Corollary 3.1. Assume that $(H 1),(H 1)$ and $(H 2)$ hold, $y_{k}(\alpha)$ is defined by Definition 2.1 with $\left\{d_{k}\right\}=\left\{y_{k}^{\prime}(0)\right\}$ satisfying (5) and (19), $\alpha_{k}$ is defined by the curve search rules (a), (b), (c), (d), (e), (f) or (g), and Algorithm Model (A) generates an infinite sequence $\left\{x_{k}\right\}$. Then

$$
\lim _{k \rightarrow+\infty}\left\|g_{k}\right\|=0 \text {. }
$$

Proof. By Theorems 3.1 and 3.2, we can complete the proof.

\section{Convergence Rate}

In order to analyze the convergence rate, we further assume that

(H3). The sequence $\left\{x_{k}\right\}$ generated by curve search method converges to $x^{*}, \nabla^{2} f\left(x^{*}\right)$ is a symmetric positive definite matrix and $f(x)$ is twice continuously differentiable on $N\left(x^{*}, \epsilon_{0}\right)=\left\{x\left\|\mid x-x^{*}\right\|<\epsilon_{0}\right\}$, where $\epsilon_{0}>0$.

Lemma 4.1. Assume that (H3) holds. Then there exist $0<m^{\prime} \leq M^{\prime}$ and $\epsilon \leq \epsilon_{0}$ such that

$$
\begin{gathered}
m^{\prime}\|y\|^{2} \leq y^{T} \nabla^{2} f(x) y \leq M^{\prime}\|y\|^{2}, \forall x, y \in N\left(x^{*}, \epsilon\right) ; \\
\frac{1}{2} m^{\prime}\left\|x-x^{*}\right\|^{2} \leq f(x)-f\left(x^{*}\right) \leq \frac{1}{2} M^{\prime}\left\|x-x^{*}\right\|^{2}, \forall x \in N\left(x^{*}, \epsilon\right) ; \\
M^{\prime}\|x-y\|^{2} \geq(g(x)-g(y))^{T}(x-y) \geq m^{\prime}\|x-y\|^{2}, \forall x, y \in N\left(x^{*}, \epsilon\right) ;
\end{gathered}
$$

and thus

$$
M^{\prime}\left\|x-x^{*}\right\|^{2} \geq g(x)^{T}\left(x-x^{*}\right) \geq m^{\prime}\left\|x-x^{*}\right\|^{2}, \forall x \in N\left(x^{*}, \epsilon\right) .
$$

By (28) and (27) we can obtain, from the Cauchy-Schwartz inequality , that

$$
M^{\prime}\left\|x-x^{*}\right\| \geq\|g(x)\| \geq m^{\prime}\left\|x-x^{*}\right\|, \forall x \in N\left(x^{*}, \epsilon\right),
$$

and

$$
\|g(x)-g(y)\| \leq M^{\prime}\|x-y\|, \forall x, y \in N\left(x^{*}, \epsilon\right) .
$$

Its proof can be seen from the book ([3], Lemma 3.1.4).

Lemma 4.2. Assume that $(H 2)$ and $(H 3)$ hold and $y_{k}(\alpha)$ is defined by Definition 2.1 and $d_{k}=y_{k}^{\prime}(0)$ satisfies (5) and (19). Algorithm (A) generates an infinite sequence $\left\{x_{k}\right\}$. Then there exist $\eta>0$ and $k^{\prime}$ such that

$$
f_{k}-f_{k+1} \geq \eta\left\|g_{k}\right\|^{2}, \forall k \geq k^{\prime}
$$

Proof. We first prove that (31) holds for the curve search rules (c), (e), (f) and (g), and then we can prove (31) also holds for the curve search rules (a), (b) and (d). 
By (9), (11), (12) and (5), we have

$$
f_{k}-f_{k+1} \geq \sigma c \alpha_{k}\left\|g_{k}\right\|^{2} .
$$

Since $x_{k} \rightarrow x^{*}(k \rightarrow \infty)$, there must exist a $k^{\prime}$ such that

$$
x_{k} \in N\left(x^{*}, \epsilon\right), \forall k \geq k^{\prime} .
$$

By (4), Cauchy Schwartz inequality and (19), we have

$$
c\left\|g_{k}\right\| \leq\left\|d_{k}\right\| \leq c_{0}\left\|g_{k}\right\| .
$$

Let

$$
\eta_{0}=\inf _{\forall k \geq k^{\prime}}\left\{\alpha_{k}\right\} .
$$

If $\eta_{0}>0$ then the conclusion is proved. If $\eta_{0}=0$, there must exist an infinite subset $K \subseteq\left\{k^{\prime}, k^{\prime}+1, k^{\prime}+2, \cdots,\right\}$ such that

$$
\lim _{k \in K, k \rightarrow \infty} \alpha_{k}=0 .
$$

Letting

$$
\alpha_{k}^{\prime}=\theta_{k} \alpha_{k} / \rho, q=1-\sigma,
$$

for (23),

$$
\alpha_{k}^{\prime}=\theta_{k} \alpha_{k}, q=\sigma,
$$

for (24) and

$$
\alpha_{k}^{\prime}=\alpha_{k}, q=1-\beta,
$$

for (13), we have

$$
\lim _{k \in K, k \rightarrow \infty} \alpha_{k}^{\prime}=0 .
$$

By (35), (23), (24), (14), (34) and Lemma 2.1, we have

$$
\begin{aligned}
\frac{c q}{c_{0}^{2}} & =\frac{c q\left\|g_{k}\right\|^{2}}{c_{0}^{2}\left\|g_{k}\right\|^{2}} \leq \frac{c q\left\|g_{k}\right\|^{2}}{\left\|d_{k}\right\|^{2}} \leq-\frac{q g_{k}^{T} d_{k}}{\left\|d_{k}\right\|^{2}} \leq \frac{\phi_{k}^{\prime}\left(\alpha_{k}^{\prime}\right)-\phi_{k}^{\prime}(0)}{\left\|d_{k}\right\|^{2}} \\
& =\frac{\left[g\left(y_{k}\left(\alpha_{k}^{\prime}\right)\right)-g_{k}\right]^{T} y_{k}^{\prime}\left(\alpha_{k}^{\prime}\right)}{\left\|d_{k}\right\|^{2}}+\frac{g_{k}^{T}\left[y_{k}^{\prime}\left(\alpha_{k}^{\prime}\right)-d_{k}\right]}{\left\|d_{k}\right\|^{2}} \\
& \leq \frac{\left(\left(y_{k}\left(\alpha_{k}^{\prime}\right)\right)-y_{k}(0)\right)^{T} \int_{0}^{1} G\left(x_{k}+t\left(y_{k}\left(\alpha_{k}^{\prime}\right)-y_{k}(0)\right)\right) \mathrm{d} t y_{k}^{\prime}\left(\alpha_{k}^{\prime}\right)}{\left\|d_{k}\right\|^{2}}+\frac{\left.\left\|g_{k}\right\| \cdot \| y_{k}^{\prime}\left(\alpha_{k}^{\prime}\right)-d_{k}\right] \|}{\left\|d_{k}\right\|^{2}} \\
& \leq \frac{\alpha_{k}^{\prime}\left(\left(\int_{0}^{1} y_{k}^{\prime}\left(t \alpha_{k}^{\prime}\right) d t\right)^{T} \int_{0}^{1} G\left(x_{k}+t\left(y_{k}\left(\alpha_{k}^{\prime}\right)-y_{k}(0)\right)\right) \mathrm{d} t y_{k}^{\prime}\left(\alpha_{k}^{\prime}\right) \mid\right.}{\left\|d_{k}\right\|^{2}}+\frac{\left.\| y_{k}^{\prime}\left(\alpha_{k}^{\prime}\right)-d_{k}\right] \|}{c\left\|d_{k}\right\|} \\
& \rightarrow 0(k \in K, k \rightarrow \infty) .
\end{aligned}
$$

The contradiction shows that $\eta_{0}=0$ does not occur and thus $\eta_{0}>0$. By letting $\eta=c \sigma \eta_{0}^{\prime}$, where

$$
\eta_{0}^{\prime}<\min \left(\eta_{0}, \frac{1}{\mathrm{~cm}^{\prime}}\right)
$$

we can obtain the conclusion.

For the curve search rules (a), (b) and (d), let $\alpha_{k}^{*}$ denote the exact step size and $\alpha_{k}$ denote the step size generated by the curve search rule (c). By the previous proof, we have 


$$
f_{k}-f\left(y_{k}\left(\alpha_{k}^{*}\right)\right) \geq f_{k}-f\left(y_{k}\left(\alpha_{k}\right)\right) \geq \eta\left\|g_{k}\right\|^{2} .
$$

All the conclusions are proved.

Theorem 4.1. Assume that (H2) and (H3) hold and $y_{k}(\alpha)$ is defined by Definition 2.1 and $d_{k}=y_{k}^{\prime}(0)$ satisfies (5) and (19). Algorithm (A) generates an infinite sequence $\left\{x_{k}\right\}$. Then $\left\{x_{k}\right\}$ converges to $x^{*}$ at least R-linearly.

Proof. By Lemmas 4.1 and 4.2 we obtain

$$
f_{k}-f_{k+1} \geq \eta\left\|g_{k}\right\|^{2} \geq \eta m^{\prime 2}\left\|x_{k}-x^{*}\right\|^{2} \geq \frac{2 \eta m^{\prime 2}}{M^{\prime}}\left(f_{k}-f^{*}\right), k \geq k^{\prime} .
$$

By setting

$$
\theta=m^{\prime} \sqrt{\frac{2 \eta}{M^{\prime}}}
$$

we can prove that $\theta<1$. In fact, by the definition of $\eta$ in the proof of Lemma 4.2 and (36), we obtain

$$
\theta^{2}=\frac{2 m^{\prime 2} \eta}{M^{\prime}} \leq \frac{2 m^{\prime 2} c \sigma \eta_{0}^{\prime}}{M^{\prime}} \leq c m^{\prime} \eta_{0}^{\prime}<1
$$

By setting

$$
\omega=\sqrt{1-\theta^{2}}
$$

and knowing $\omega<1$, we obtain from the above inequality that

$$
\begin{aligned}
f_{k+1}-f^{*} & \leq\left(1-\theta^{2}\right)\left(f_{k}-f^{*}\right) \\
& =\omega^{2}\left(f_{k}-f^{*}\right) \\
& \leq \cdots \\
& \leq \omega^{2\left(k-k^{\prime}\right)}\left(f_{k^{\prime}+1}-f^{*}\right) .
\end{aligned}
$$

By Lemma 4.1 and the above inequality we have

$$
\begin{aligned}
\left\|x_{k+1}-x^{*}\right\|^{2} & \leq \frac{2}{m^{\prime}}\left(f_{k+1}-f^{*}\right) \\
& \leq \omega^{2\left(k-k^{\prime}\right)} \frac{2\left(f_{k^{\prime}+1}-f^{*}\right)}{m^{\prime}},
\end{aligned}
$$

thus

$$
\left\|x_{k+1}-x^{*}\right\| \leq \omega^{k-k^{\prime}} \sqrt{\frac{2\left(f_{k^{\prime}+1}-f^{*}\right)}{m^{\prime}}} .
$$

i.e.,

$$
\left\|x_{k}-x^{*}\right\| \leq \omega^{k} \sqrt{\frac{2\left(f_{k^{\prime}+1}-f^{*}\right)}{m^{\prime} \omega^{2\left(k^{\prime}+1\right)}} .}
$$

Therefore,

$$
R_{1}\left\{x_{k}\right\}=\lim _{k \rightarrow \infty}\left\|x_{k}-x^{*}\right\|^{\frac{1}{k}} \leq \omega,
$$

which shows that $\left\{x_{k}\right\}$ converges to $x^{*}$ at least R-linearly. 


\section{Some Implementable Version}

\subsection{How to Find Curves}

In order to find some curves satisfying Definition 2.1 and $(H 2)$, we first investigate the slope and curvature of a curve. Given a curve $x=y_{k}(\alpha)$ satisfying $y_{k}(0)=x_{k}$, if it is twice continuously differentiable, then the slope of the curve at $\alpha=0$ is $y_{k}^{\prime}(0)$ and the curvature is $y_{k}^{\prime \prime}(0)$. We hope that the curve is a descent curve at $\alpha=0$, i.e. $y_{k}^{\prime}(0)^{T} g_{k}<0$. Generally, we require $y_{k}^{\prime}(0)$ to satisfy

$$
y_{k}^{\prime}(0)^{T} g_{k} \leq-c\left\|g_{k}\right\|^{2},\left\|y_{k}^{\prime}(0)\right\| \leq c_{0}\left\|g_{k}\right\|,
$$

where $0<c \leq 1$ and $c_{0}>1$. Moreover, we expect the curves to satisfy

$$
\left\|y_{k}^{\prime}(\alpha)\right\| \leq M,\left\|y_{k}^{\prime \prime}(\alpha)\right\| \leq \bar{M}\left\|y_{k}^{\prime}(0)\right\|, \forall k .
$$

It is worthy to point out that many convergence properties of curve search methods remain hold for line search method. In fact, the line $y_{k}(\alpha)=x_{k}+\alpha d_{k}$ satisfies Definition 2.1 and (H2), provided that $\left\{d_{k}\right\}$ is bounded for $k \geq 0$. For example, we take $y_{k}(\alpha)=x_{k}+\alpha d_{k}(\alpha)$ with

$$
d_{k}(\alpha)= \begin{cases}-g_{k}, & \text { if } k \leq m-1 \\ -\left(\left(1-\sum_{i=2}^{m} \alpha^{i-1} s_{k}^{i}\right) g_{k}+\sum_{i=2}^{m} \alpha^{i-1} s_{k}^{i} d_{k-i+1}\right), & \text { if } k \geq m,\end{cases}
$$

where $m$ is a positive integer and

$$
s_{k}^{i}=\frac{\rho\left\|g_{k}\right\|^{2}}{(m-1)\left[\left\|g_{k}\right\|^{2}+\left|g_{k}^{T} d_{k-i+1}\right|\right]}, i=2,3, \cdots, m .
$$

We can prove that $y_{k}(\alpha)$ satisfies Definition 2.1 and $(H 2)$ under some mild conditions. Numerical results showed that the curve search method was more efficient than some line search methods [16].

Another curve search method is from [15] with the curve defined by $y_{k}(\alpha)=x_{k}+\alpha d_{k}(\alpha)$ where

$$
d_{k}(\alpha)= \begin{cases}-g_{k}, & \text { if } k=1 \\ -\left[\left(1-\frac{\alpha s_{k}}{1+\alpha}\right) g_{k}+\frac{\alpha s_{k}}{1+\alpha} d_{k-1}\right], & \text { if } k \geq 2\end{cases}
$$

and

$$
S_{k}= \begin{cases}1, & \text { if } k=1 \\ \left\|g_{k}\right\|^{2} /\left[\left\|g_{k}\right\|^{2}+\left|g_{k}^{T} d_{k-1}\right|\right], & \text { if } k \geq 2 .\end{cases}
$$

This curve also satisfies Definition 2.1 and (H2) with $y_{k}^{\prime}(0)=d_{k}$ satisfying (5) under certain conditions and has good numerical performance.

Moreover, many researchers take

$$
y_{k}(\alpha)=x_{k}+\alpha d_{k}+\frac{1}{2} \alpha^{2} z_{k}
$$

and $d_{k}=y_{k}^{\prime}(0)$ satisfies (4) [20]. Certainly, we can obtain some curves by solving initial problems or boundaryvalue problems of ordinary differential equations and sometimes by using interpolation technique. Lucidi, Ferris and Roma proposed a curvilinear truncated Newton method which uses the curve

$$
y_{k}(\alpha)=x_{k}+\alpha^{2} s_{k}+\alpha d_{k},
$$

with $s_{k}$ being the quasi-Newton direction and $d_{k}$ being the steepest descent direction. This method also has good numerical performance [18] [19] because it reduces to quasi-Newton method finally and avoids some disadvantages of quasi-Newton method at the initial iterations. We guess that there may be many curve search methods which are superior to line search methods in numerical performance.

For example, if we take $d_{k}=-g_{k}$ and suppose that $\left\{q_{k}\right\}$ and $\left\{p_{k}\right\}$ are uniformly bounded for $k$, then the 
following curve

$$
y_{k}(\alpha)=x_{k}-\alpha_{k} g_{k}+\frac{1}{2} \alpha^{2}\left\|g_{k}\right\| p_{k}+\frac{1}{6} \alpha^{3}\left\|g_{k}\right\| q_{k}
$$

satisfies (H2), provided that $\left\{g_{k}\right\}$ is bounded. In the following we shall test some curve search methods.

\subsection{Numerical Experiments}

In this subsection, some numerical reports are prisented for some implementable curve search methods. First of all, we consider some curve search methods with memory gradients. The first curve search method is based on the curve

$$
y_{k}(\alpha)=x_{k}-\alpha\left[(1-\alpha) g_{k}+\alpha\left\|g_{k}\right\| g_{k-1}\right], k \geq 1,
$$

The second curve search method is to use the curve

$$
y_{k}(\alpha)=x_{k}-\alpha\left[(1-\alpha) g_{k}+\alpha\left\|g_{k}\right\| d_{k-1}\right], k \geq 1,
$$

and the third curve search method searches along the curve at each iteration

$$
y_{k}(\alpha)=x_{k}-\alpha_{k} g_{k}+\frac{1}{2} \alpha^{2}\left\|g_{k}\right\| g_{k-1}+\frac{1}{6} \alpha^{3}\left\|g_{k}\right\| g_{k-2}, k \geq 2 .
$$

We use respectively the Armijo curve search rule and the Wolfe curve search rule to the above three curves to find a step size at each step. Test problems 21 - 35 and their initial iterative points are from the literature [21]. For example, Problem 21 stands for the problem 21 in the literature and so on.

In the curve search rules (c) and (g) we set the parameters $\sigma=0.25, \rho=0.75, L=1$ and $\beta=0.38$. Numerical performance of the three curve search methods is reported in Table 1 and a pair of numbers means that the first number denotes the number of iterations and the second number denotes the number of functional evaluations. "P" stands for problems, $n$ is the dimension of problems and T denotes total CPU time for solving

\begin{tabular}{|c|c|c|c|c|c|c|c|}
\hline $\mathrm{P}$ & $\mathrm{n}$ & $\mathrm{A} 1$ (c) & A1(g) & A2(c) & A2(g) & A3(c) & A3(g) \\
\hline 21 & $10^{4}$ & 193/1089 & $118 / 673$ & $168 / 1982$ & 132/982 & $145 / 869$ & $156 / 1421$ \\
\hline 22 & $10^{4}$ & $254 / 2736$ & $212 / 1983$ & $316 / 1572$ & $247 / 2195$ & $231 / 1673$ & 238/1965 \\
\hline 23 & $10^{4}$ & $121 / 689$ & $128 / 513$ & $98 / 1034$ & 122/832 & $117 / 968$ & $146 / 872$ \\
\hline 24 & $10^{4}$ & $316 / 1863$ & $235 / 1493$ & 356/1987 & $234 / 1392$ & $198 / 1326$ & $168 / 1628$ \\
\hline 25 & $10^{4}$ & $119 / 628$ & 121/892 & $126 / 916$ & $115 / 1639$ & $179 / 1473$ & $105 / 1034$ \\
\hline 26 & $10^{4}$ & $178 / 2134$ & $192 / 2075$ & 169/1935 & $142 / 1432$ & $128 / 1732$ & $126 / 1728$ \\
\hline 27 & $10^{4}$ & 127/982 & $134 / 763$ & $133 / 1772$ & $152 / 1827$ & $109 / 913$ & $118 / 1471$ \\
\hline 28 & $10^{4}$ & $153 / 918$ & $217 / 1528$ & $145 / 1463$ & $143 / 1367$ & $135 / 1731$ & $129 / 1862$ \\
\hline 29 & $10^{4}$ & $183 / 2156$ & 137/1985 & $163 / 3721$ & $169 / 2176$ & $165 / 2191$ & 127/1632 \\
\hline 30 & $10^{4}$ & $152 / 962$ & 123/1891 & $106 / 2732$ & $136 / 1472$ & $145 / 1569$ & $113 / 1528$ \\
\hline 31 & $10^{4}$ & $117 / 1465$ & 109/1394 & $127 / 1528$ & $137 / 1647$ & $134 / 1841$ & $152 / 1378$ \\
\hline 32 & $10^{4}$ & $98 / 1275$ & $126 / 1763$ & 129/972 & 104/1166 & $111 / 1634$ & 94/982 \\
\hline 33 & $10^{4}$ & 129/863 & 116/1872 & $162 / 1798$ & $181 / 1744$ & $148 / 1825$ & $116 / 1872$ \\
\hline 34 & $10^{4}$ & 67/862 & $95 / 962$ & 86/739 & 74/763 & $88 / 1267$ & $85 / 1621$ \\
\hline 35 & $10^{4}$ & $432 / 3721$ & $269 / 2964$ & $195 / 1267$ & $342 / 2374$ & 253/1288 & $317 / 1268$ \\
\hline $\mathrm{T}$ & - & $653 \mathrm{~s}$ & $436 s$ & 548s & 463s & 553s & $414 \mathrm{~s}$ \\
\hline
\end{tabular}
all the 15 problems. We denote A1, A2 and A3 the curve search methods with the curves (40), (41) and (42) respectively. $\mathrm{A} 1(\mathrm{c})$ and $\mathrm{A} 1(\mathrm{~g})$ means the $\mathrm{A} 1$ algorithm with the curve search rule (c) and the $\mathrm{A} 1$ algorithm with

\section{Table 1. Iterations and function evaluations.}


the curve search rule (g) respectively, and so on. The stop criteria is

$$
\left\|g_{k}\right\| \leq 10^{-8} \text {. }
$$

It is shown in Table 1 that curve search methods with memory gradients converge to the optimal solutions stably and averagely. In addition, curve search methods with the Wolfe curve search rule are superior to the methods with the Armijo curve search rule. This shows that $L=1$ seems to be an inadequate choice in the Armijo curve search rule and we can take $L$ variably at each step similarly as in the literature [16].

Moreover, many line search methods may fail to converge when solving some practical problems, especially when solving large scale problems, while curve search methods with memory gradients always converge stably. From this point of view, we guess that some curve search methods are available and promising for optimization problems.

\section{Conclusions}

Some curve search methods have good numerical performance and are superior to the line search methods to certain extent. This motivates us to investigate the general convergence properties of these promising methods.

In this paper we presented a class of curve search methods for unconstrained minimization problems and proved its global convergence and convergence rate under some mild conditions. Curve search method is a generalization of line search methods but it has wider choices than line search methods. Several curve search rules were proposed and some approaches to choose the curves were presented. The idea of curve search methods enables us to find some more efficient methods for minimization problems. Furthermore, numerical results showed that some curve search methods were stable, available and efficient in solving some large scale problems.

For the future research, we should investigate more techniques for choosing search curves that contain the information of objective functions and find more curve search rules for the curve search method.

\section{References}

[1] Vrahatis, M.N., Androulakis, G.S., Lambrinos, J.N. and Magoulas, G.D. (2002) A Class of Gradient Unconstrained Minimization Algorithms with Adaptive Stepsize. Journal of Computational and Applied Mathematics, 114, 367-386. http://dx.doi.org/10.1016/S0377-0427(99)00276-9

[2] Nocedal, J. and Wright, J.S. (1999) Numerical Optimization. Springer-Verlag New York, Inc., New York. http://dx.doi.org/10.1007/b98874

[3] Yuan, Y.X. (1993) Numerical Methods for Nonlinear Programming. Shanghai Scientific \& Technical Publishers, Shanghai.

[4] McCormick, G.P. (1975) An Arc Method for Nonlinear Programming. SIAM Journal on Control, 13, 1194-1216. http://dx.doi.org/10.1137/0313075

[5] Zang, I. (1978) A New Arc Algorithm for Unconstrained Optimization. Mathematical Programming, 15, 36-52. http://dx.doi.org/10.1007/BF01608998

[6] Botsaris, C.A. (1978) Differential Gradient Methods. Journal of Mathematical Analysis and Applications, 63, 177-198. http://dx.doi.org/10.1016/0022-247X(78)90114-2

[7] Botsaris, C.A. (1978) A Curvilinear Optimization Method Based upon Iterative Estimation of the Eigensystem of the Hessian Matrix. Journal of Mathematical Analysis and Applications, 63, 396-411. http://dx.doi.org/10.1016/0022-247X(78)90085-9

[8] Botsaris, C.A. (1978) A Class of Methods for Unconstrained Minimization Based on Stable Numerical Integration Techniques. Journal of Mathematical Analysis and Applications, 63, 729-749. http://dx.doi.org/10.1016/0022-247X(78)90068-9

[9] Botsaris, C.A. and Jacobson, D.H. (1976) A Newton-Type Curvilinear Search Method for Optimization. Journal of Mathematical Analysis and Applications, 54, 217-229. http://dx.doi.org/10.1016/0022-247X(76)90246-8

[10] Flam, S.D. (1992) Solving Convex Programming by Means of Ordinary Differential Equations. Mathematics of Operations Research, 17, 290-302. http://dx.doi.org/10.1287/moor.17.2.290

[11] Syman, J.A. (1982) A New and Dynamic Method for Unconstrained Minimization. Applied Mathematical Modelling, 6, 449-462. http://dx.doi.org/10.1016/S0307-904X(82)80007-3

[12] Schropp, J. (1997) A Note on Minimization Problems and Multistep Methods. Numerische Mathematik, 78, 87-101. 
http://dx.doi.org/10.1007/s002110050305

[13] van Wyk, D.J. (1984) Differential Optimization Techniques. Applied Mathematical Modelling, 8, 419-424. http://dx.doi.org/10.1016/0307-904X(84)90048-9

[14] Wu, X.Y., Xia, J.L. and Ouyang, Z.X. (2002) Note on Global Convergence of ODE Methods for Unconstrained Optimization. Applied Mathematics and Computation, 125, 311-315. http://dx.doi.org/10.1016/S0096-3003(00)00133-8

[15] Shi, Z.J. and Shen, J. (2005) A New Descent Algorithm with Curve Search Rule. Applied Mathematics and Computation, 161, 753-768. http://dx.doi.org/10.1016/j.amc.2003.12.058

[16] Shi, Z.J. (2004) Convergence of Multi-Step Curve Search Method for Unconstrained Optimization. Journal of Numerical Mathematics, 12, 297-309. http://dx.doi.org/10.1515/1569395042571292

[17] Amaya, J. (1985) On the Convergence of Curvilinear Search Algorithms in Unconstrained Optimization. Operations Research Letters, 4, 31-34. http://dx.doi.org/10.1016/0167-6377(85)90048-3

[18] Ferris, M., Lucidi, S. and Roma, M. (1996) Nonmonotone Curvilinear Line Search Methods for Unconstrained Optimization. Computational Optimization and Applications, 6, 117-136. http://dx.doi.org/10.1007/BF00249642

[19] Lucidi, S. and Roma, M. (1997) Numerical Experiences with New Truncated Newton Methods in Large Scale Unconstrained Optimization. Computational Optimization and Applications, 7, 71-87. http://dx.doi.org/10.1023/A:1008619812615

[20] Ben-Tal, A., Melman, A. and Zowe, J. (1990) Curve Search Methods for Unconstrained Optimization. Optimization, 21, 669-695. http://dx.doi.org/10.1080/02331939008843594

[21] Moré, J.J., Garbow, B.S. and Hillstrom, K.H. (1981) Testing Unconstrained Optimization Software. ACM Transactions on Mathematical Software, 7, 17-41. http://dx.doi.org/10.1145/355934.355936 